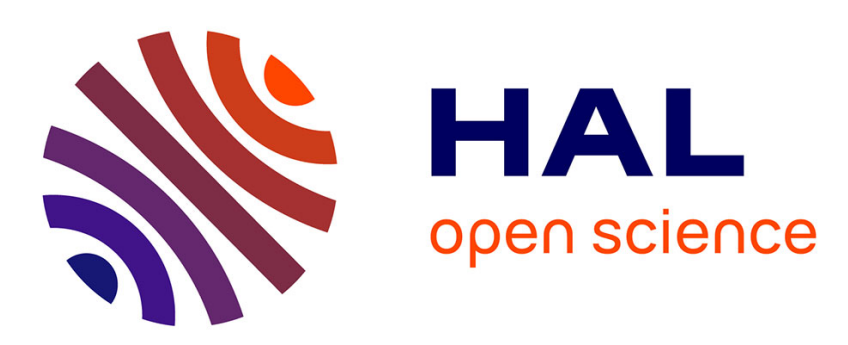

\title{
Magneto-Optical Study of the Anisotropic Piezoelectric Effect in a Ferroelectric Crystal
}

\author{
A. Klimov, Nicolas Tiercelin, Vladimir Preobrazhensky, A. Sigov, Philippe \\ Pernod
}

\section{- To cite this version:}

A. Klimov, Nicolas Tiercelin, Vladimir Preobrazhensky, A. Sigov, Philippe Pernod. Magneto-Optical Study of the Anisotropic Piezoelectric Effect in a Ferroelectric Crystal. Bulletin of the Russian Academy of Sciences - Physics, 2019, 83 (7), pp.888-890. 10.3103/S1062873819070207 . hal-02318296

\author{
HAL Id: hal-02318296 \\ https://hal.science/hal-02318296
}

Submitted on 15 Oct 2020

HAL is a multi-disciplinary open access archive for the deposit and dissemination of scientific research documents, whether they are published or not. The documents may come from teaching and research institutions in France or abroad, or from public or private research centers.
L'archive ouverte pluridisciplinaire HAL, est destinée au dépôt et à la diffusion de documents scientifiques de niveau recherche, publiés ou non, émanant des établissements d'enseignement et de recherche français ou étrangers, des laboratoires publics ou privés. 


\title{
Magneto-Optical Study of the Anisotropic Piezoelectric Effect in a Ferroelectric Crystal
}

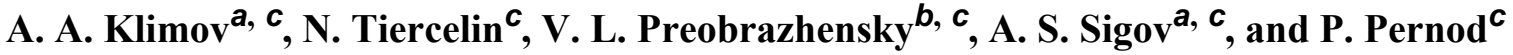 \\ Joint International Laboratory LIA LICS:

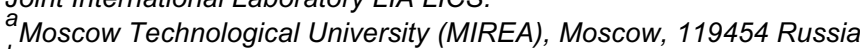 \\ ${ }^{b}$ Prokhorov General Physics Institute, Russian Academy of Sciences, Moscow, 117942 Russia \\ ${ }^{C}$ CNRS, Centrale Lille, Univ. Lille, Univ. Polytechnique Hauts-de-France, UMR 8520 - IEMN, F-59000 Lille, France
}

\begin{abstract}
The possibility of using the magneto-optical Kerr effect in thin magnetostrictive films to study the piezoelectric characteristics of ferroelectrics is demonstrated experimentally and theoretically. A 011 PMN-PT ferroelectric crystal with an $\mathrm{N}^{*}\left(\mathrm{TbCo}^{2} / \mathrm{FeCo}\right)$ magnetostrictive nanostructure deposited on its surface is the object of study.
\end{abstract}

\section{INTRODUCTION}

Numerous studies of the effect deformation has on the physical properties of materials and structures have in recent years created a promising line of solid-state electronics referred to generically as straintronics[1]. Artificial multiferroics composed of elastically attached (strain-mediated) magnetostrictive and piezoelectric components are a striking example of using the straintronics concept to create materials with new properties. These materials are characterized by strong magnetoelectric coupling, which is of consider-able interest for different technical applications. Magnetoelectric memory with random access (MEL-RAM) and ultralow energy consumption is one intensely studied application [2, 3]. The basis for elements of this memory is heterostructures that contain magnetostrictive films or nanoparticles with bi- or multistable magnetic systems, deposited on ferroelectric substrates. A key role in the emergence of magnetoelectric interaction in these heterostructures is played by the anisotropic piezoelectric effect in a ferroelectric, which creates deformation in a magnetic film; the symmetry of the former allows the switching of magnetization between different stable states [4]. To study the piezoelectric effect experimentally, research-ers generally employ either resistive transducers, glued to the samples of interest, or ultrasonic and resonance acoustic means. These approaches are difficult to use with samples that are small or have complex topologies, which are of interest for straintronic applications. In this work, the principle of the local study of piezo-electric deformations is demonstrated using the mag neto-optic Kerr effect (MOKE) in a magnetostrictive film deposited on the surface of a ferroelectric.

\section{PRINCIPLE AND RESULTS OF MAGNETO-OPTICAL MEASUREMENTS OF PIEZODEFORMATION}

A $300-\mu$ m-thick PMN-PT ferroelectric crystal with a 011 cut and an electrode deposited on its bottom surface was our object of study for piezodeformations. A $20 *\left(\mathrm{TbCo}_{25 \mathrm{~nm}} / \mathrm{FeCo}_{5 \mathrm{~nm}}\right)$ nanostructured magnetostrictive film acting as both a sensor and a top electrode was deposited onto the top surface of the crystal via cathode sputtering in a magnetic field [4, 5]. Uniaxial magnetic anisotropy with an axis of easy magnetization oriented at an angle of $45^{\circ}$ to the 100 PMN-PT crystallographic axis was induced in the film by selecting the direction of the magnetic field during sputtering. The structure was placed into magnetic field $\vec{H}$, which was perpendicular to the axis of easy magnetization (EA), as is shown in the schematic in Fig. 1. The structural geometry was similar to the one used in $[3,4]$ to demonstrate the magnetoelectric recording of information by a pulsed electric field.

The energy density of the magnetic subsystem as a function of angle $\phi$ of the orientation of magnetization vector $\vec{M}$ had the form :

$$
\begin{aligned}
F_{\mathrm{m}}= & -M H \cos \varphi-\frac{1}{2} M H_{\mathrm{A}} \sin ^{2} \varphi \\
& +\frac{1}{2} B\left(u_{x x}-u_{y y}\right) \sin 2 \varphi,
\end{aligned}
$$


where $\mathrm{B}$ is the constant of magnetostriction; $\mathrm{H}_{\mathrm{A}}$ is the strength of the anisotropy field; and uij is the tensor of piezo-deformation created in the film by the piezo substrate when electric voltage is applied to the electrodes. The magnetic system becomes bistable when magnetizing the film with magnetic field $\mathrm{H}<\mathrm{H}_{\mathrm{A}}$. This is used for the binary recording of information with pulses of the electric field applied to the substrate. Under the strength of a magnetizing field that exceeds that of anisotropy field $\mathrm{H}>\mathrm{H}_{\mathrm{A}}$, the magnetic system changes to the saturation state with magnetization directed along $\vec{H}$. Deformation of the film is accompanied by the magnetization deviating from the direction of the field. We then observe a certain relationship between the deformation and the normalized projection of magnetization on the easy axis: $\mathrm{m}_{\xi}=\mathrm{M}_{\xi} / \mathrm{M}$. Thus,

$$
u_{x x}-u_{y y}=\frac{M H_{\mathrm{A}}}{B} \frac{m_{\xi}\left(h-\sqrt{1-m_{\xi}^{2}}\right)}{\left(1-2 m_{\xi}^{2}\right)},
$$

where $h=H / H_{\mathrm{A}}$. The projection of magnetization can be registered without contact using a meridional MOKE, where light propagates in a plane parallel to the easy axis. To use relation (2) in calculating the deformation, the MOKE signal must be normalized to the magneto- optical signal measured when magnetizing the film to saturation along the axis of anisotropy. Due to the thinness the film compared to the thick- ness of the crystal, an anisotropic deformation measured in this manner does not differ from the proper piezo-deformation of a ferroelectric.

The sensitivity of the magneto-optical approach to registration increases near the point of spin reorientation $H \rightarrow H_{\mathrm{A}}$. Figure 2 illustrates the increase in a MOKE signal generated by a weak alternating electric voltage at $H \rightarrow H_{\mathrm{A}}=1.4 \mathrm{kOe}$. Results from magneto-optical measurements of the dependence of magnetization projection $\mathrm{m}_{\xi}(U)$ onto the easy axis on electric voltage $U$ applied to a sample are given in Fig. 3a. The sensitivity of the MOKE signal to the electric field grows as the magnetizing field approaches the field of anisotropy. Figure $3 \mathrm{~b}$ shows the result from recalculating dependences $m_{\xi}(U)$ to the dependence of anisotropic deformation $u_{x x}-u_{y y}$ on voltage $U$ using the known parameters of the magnetic film: $M=800 \mathrm{G}$ and $B=10 \mathrm{MPa}$.

Our data demonstrate the hysteresis behavior of an anisotropic piezoelectric effect with a coercive force of around $17 \mathrm{kV} / \mathrm{m}$ in relatively weak applied fields $E_{\max }=0.1 \mathrm{MV} / \mathrm{m}$. The full range of the change in deformation agrees with the data of [6], obtained for PMN-PT $\langle 011\rangle$ crystals using resistive strain-gauge transducers. At the same time, the coercivity of the sample we studied was appreciably lower in weak fields. Individual cycles of the hysteresis loops presented in Fig. 3b correspond to anisotropic piezoelectric moduli $d 31^{-} d_{32}=6 \times 10^{-9} \mathrm{C} / \mathrm{N}$, which corresponds in order of magnitude to the value obtained in [7] by ultrasound means. The structural sensitivity of the crystal parameters and their considerable dependence on specific conditions of prepolarization for individual PMN-PT samples could be the reason for observed quantitative differences.

\section{CONCLUSIONS}

The sputtering of intermetallic magnetostrictive nanostructures imposes no specific requirements on the materials of a substrate. The straintronic magneto- optical study of deformations described in this work is thus suitable for a wide range of piezoelectric sub- stances. Modern lithography can deposit strain-sensitive magneto-optical films onto topologically complex structures and elements of devices. Films used as elements for the local control of deformations can in this case also act as metallic electrodes. Finally, the range of applicability of the magneto-optical study of deformation is not restricted to piezoelectric materials, and can be used in different fields of materials science. Use of the MOKE for visualizing elastic stresses in nonmagnetic metals was described independently in [8]. 


\section{FUNDING}

This work was supported by the Russian Foundation for Basic Research, project no. 16-29-14022.

\section{REFERENCES}

1. Bukharaev, A.A., Zvezdin, A.K., Pyatakov, A.P., and Fetisov, Yu.K., Phys.-Usp., 2018, vol. 61, p. 1175.

2. Nanomagnetic and Spintronic Devices for Energy-Effi- cient Memory and Computing, Atulasimha, J. and Bandyopadhyay, S., Eds., Wiley, 2016.

3. Klimov,A., Tiercelin, N.,Dusch,Y., etal., Appl.Phys. Lett., 2017, vol. 110, no. 22, p. 222401.

4. Dusch, Y., Tiercelin, N., Klimov, A., et al., J. Appl. Phys., 2013, vol. 113, no. 17, p. 17C719.

5. Klimov, A., Tiercelin, N., Preobrazhensky, V., and Pernod, P., IEEE Trans. Magn., 2006, vol. 42, no. 10 , p. 3090.

6. Wu, T., Zhao, P., Bao, M., et al., J. Appl. Phys., 2011, vol. 109, p. 124101.

7. Wang, F., Luo, L., and Zhou, D., Appl. Phys. Lett., 2007, vol. 90, p. 212903.

8. Jourdan, M., Krämer, M.M.B., Kläui, M., et al., J. Magn. Magn. Mater., 2018, vol. 465, p. 143.

\section{Figures}

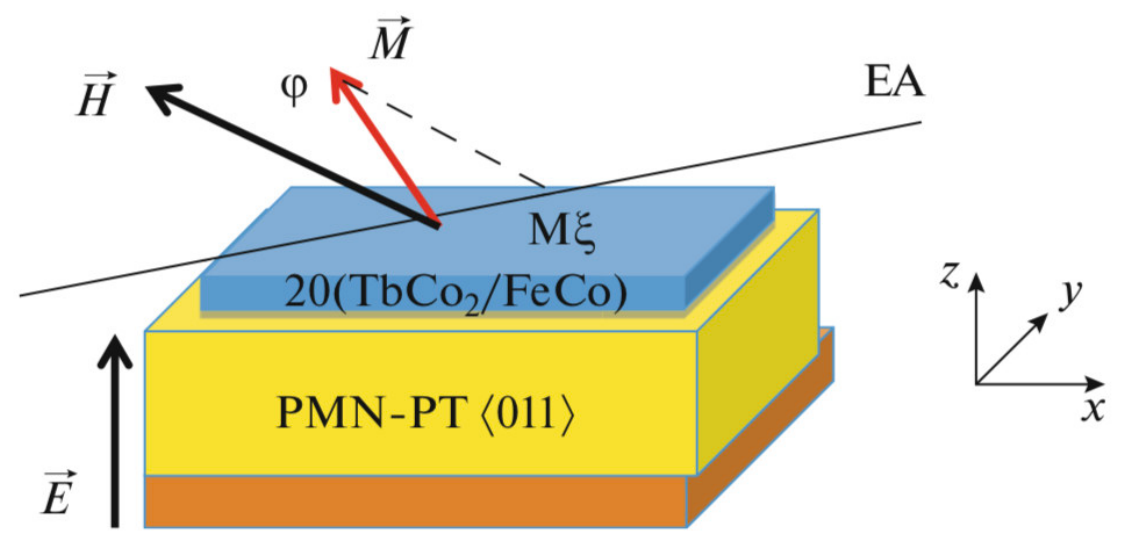

Fig. 1. Geometry of the experiment for our magneto-opti- cal study of anisotropic piezoelectric deformations in a PMNPT crystal. Easy magnetization axis EA is oriented at an angle of $45^{\circ}$ to the $\langle 100\rangle$ PMN-PT crystallographic axis.

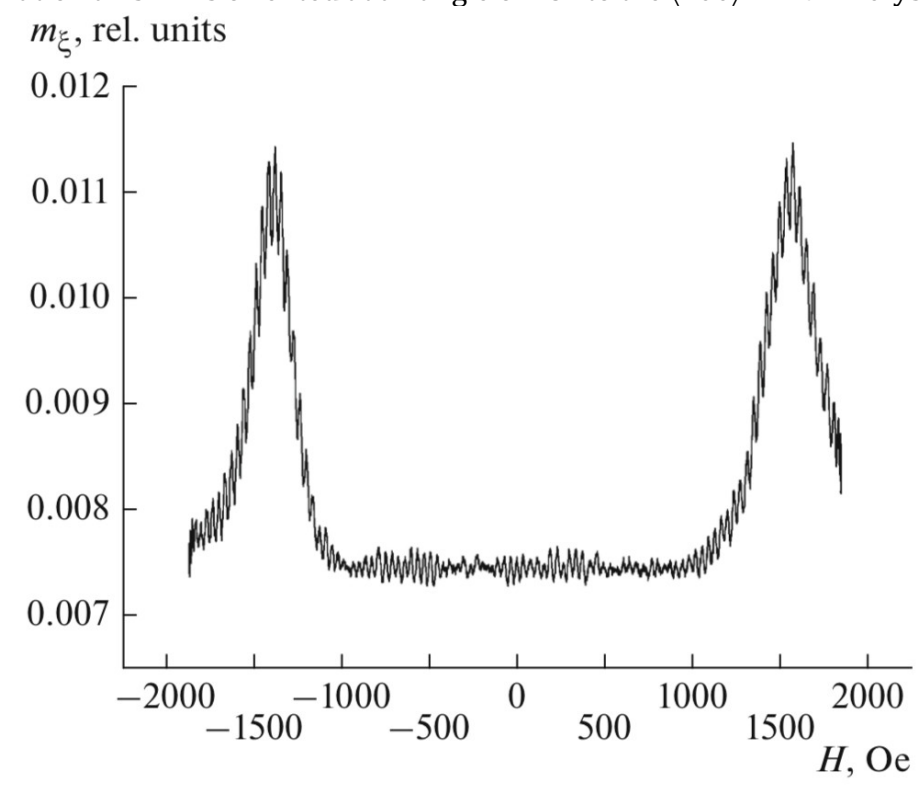

Fig. 2. Dependence of the amplitude of a magneto-optical signal on the magnetic field strength. The signal is induced by alternating electric voltage applied to a PMN-PT crystal. 
(a)



(b)



Fig. 3. (a) Measured with the MOKE dependence of the normalized projection of a magnetic moment on easy axis EA on the electric voltage at different strengths of a trans- verse magnetizing field; (b) anisotropic piezoelectric deformations, calculated using relation (2) with the data from magneto-optical measurements (a). 